\title{
Modeling dynamics of the spacecraft power plant consisting of Stirling engine and external thermal circuit
}

\author{
Aigiz Valiullin ${ }^{1, *}$, Vladimir Zenkin ${ }^{1}$ \\ ${ }^{1}$ Bauman Moscow State Technical University, Moscow, Russia, 105005
}

\begin{abstract}
Modern space programs cover a wide range of missions, including both near-earth missions and those affecting areas of deep space. In the second case, power plants based on the conversion of thermal energy into electrical energy are often considered for the power supply of spacecraft. One of the possible thermoelectric converters is the Stirling engine. To be able to design modern power plants of spacecraft built based on Stirling engines, it is necessary to use calculation tools to simulate their operation in a wide range of modes, to take into consideration the influence of subsystems on each other and the final characteristics of the power plant. As part of the work, a thermodynamic model of an external thermal circuit was created, consisting of heat pipes, heat exchangers and radiator cooler panels. The hot thermal circuit is consisted of two parts: primary and secondary, the cold thermal circuit is consisted of only one part. A mathematical model of the free-piston Stirling engine of the first order has been also implemented. The working fluid in the engine is helium. The developed models were independently debugged and then integrated into a single model of the power plant. For each type of model, the corresponding mathematical models are presented and the basic assumptions are described. The model is implemented in an open simulation environment of xcos/scilab, examples of power plant model implementation are presented. Numerical experiments were carried out to study the dynamics of the heating of the contour elements (with independent, and then with integrated modeling) and the effect of the non-stationary thermal state on the engine performance. The article presents the basic equations and the design schemes for the power plant model, as well as some results of numerical simulation, including the dynamics of hot and cold circuit temperature changes and the dynamics of changes in the indicator diagram of the engine when the external thermal circuit is heated. The developed model can be used in the early stages of spacecraft design for a preliminary assessment of the main indicators of the power plant, its dimensions and thermal loads.
\end{abstract}

* Corresponding author: valiullin.aigiz@yandex.ru 


\section{Introduction}

Modern space programs cover a wide range of different missions. They include both tasks in near-Earth orbit and tasks affecting areas of deep space. Providing the spacecraft with energy is one of the main tasks that must be solved to complete the mission. Although the use of solar panels is widespread, in some cases a power plant built on their basis is irrational. This applies to missions on distant planets where solar radiation is not enough to provide energy. Also, solar panels are irrational in a number of planetary missions, for which spacecraft require high power. In such cases other types of power installations are considered for the power supply of spacecraft, including those based on the conversion of thermal energy into electrical energy.

One of the possible thermal-electric converters is the Stirling engine [1, 2, 3]. As a rule, first of all it's free-piston version is considered, which is known as the Beale engine. Power plants built on the basis of the Stirling engine have a number of advantages: a sufficiently high efficiency (up to $30 \%$ ), a long service life (up to 120,000 hours) $[4,5]$, the ability to work from any heat source (including solar radiation, a nuclear reactor, etc.). Thus, the US NASA has funded several large programs for the development of power plants based on Stirling engines [3].

Within the framework of modern methods of designing technical devices, methods of computational analysis are of great importance. They allow you to determine the necessary parameters of the device components at the early stages of design, evaluate their interaction with each other, etc. For spacecraft power plants, this task is divided into two parts: 1) modelling of a Stirling free-piston engine; 2) integration of the engine model with models of auxiliary systems, including an external thermal circuit, an electrical load and a control system.

A large number of researches are devoted to modelling Stirling engines for various purposes, such as the works of Martini, Walker, Finkelstein and Schmidt [1, 2, 6]. At the moment, a hierarchy of mathematical models has been formed [2,6], ranging from thermodynamic to spatial CFD models. Depending on the tasks to be solved, all these models find their application. As a rule, simple models of the first or second order are used for modelling a power plant [7]. Examples of such models can be found in publications [8, $9,10,11,12]$, where they are used for dynamic analysis of the Stirling self-oscillatory system, analysis of its stability and selection of parameters of the oscillatory system.

The work of the entire energy system as a whole also attracts the attention of engineers. Thus, in [13], a simulation of a power plant with a nuclear reactor was carried out and the dynamics of the system in various emergency situations was considered. In the works of Regan, T. and Levandovsky [14, 15], a joint simulation of the Stirling engine and its electrical load was carried out. The model takes into account the influence of the nonstationary thermal state of the external thermal circuit, and the electrical load ensures stable operation of the installation by adjusting its electrical components.

The creation of an integrated model of the power system is aimed at creating a tool that provides the ability to predict the behavior of the system, changes in its useful properties in the conditions of complex interaction of its subsystems, and interaction with the environment. This provides a potential opportunity to calculate the overall dynamics of the system, optimize the parameters in real operating conditions, as well as assess the environmental impact and possible breakdowns.

In the application to space technology, for the development of modern energy systems of spacecraft, it is necessary to create a mathematical model of the power plant, to evaluate its dynamics and effective indicators. In order to achieve this goal, the task was to create a model of power plant consisting free-piston Stirling engine and external thermal circuit consisting of heat pipes, heat exchangers and radiator cooling panels. 


\section{Methods}

\subsection{Stirling engine model}

For the computational analysis of a free piston Stirling engine, a first order thermodynamic model was developed. The installation developed by NASA was chosen as the basis [13]. The working body is helium. Working frequency is $60 \mathrm{~Hz}$. The design scheme is shown in Figure 1.

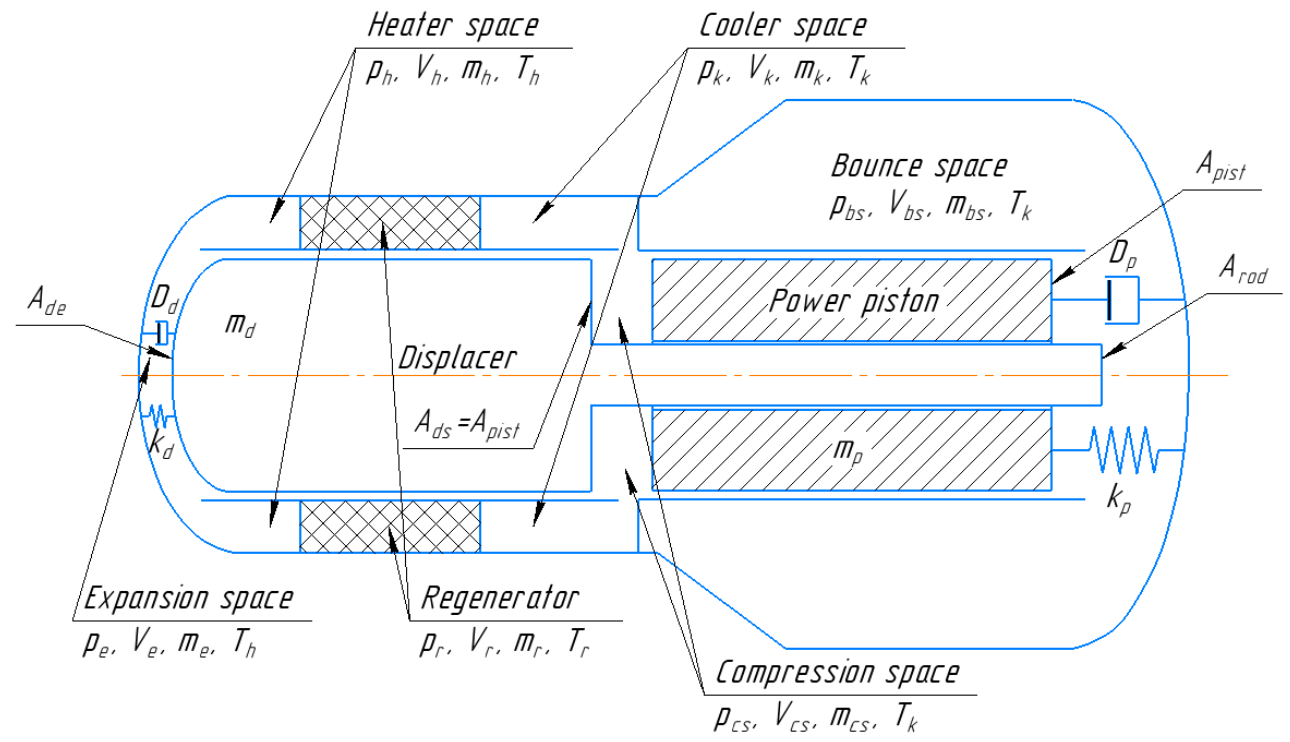

Fig. 1. Design scheme of the Stirling engine.

\subsubsection{Mathematical model}

When formulating the mathematical model of the Stirling engine, a number of assumptions were made:

- The working area is divided into 6 volumes: compression space $\left(V_{c s}\right)$, cooling area $\left(V_{k}\right)$, regenerator $\left(V_{r}\right)$, heating area $\left(V_{h}\right)$, expansion space $\left(V_{e}\right)$, buffer space $\left(V_{b}\right)$;

- Constant temperature in the spaces during the cycle;

- Equality of pressures in the spaces (changes with time during the cycle);

- There are no leaks through the gaps;

- The gas temperature in the heating and cooling areas, respectively, is equal to the temperatures of the heater and cooler.

Due to the absence of leaks, the mass of the working fluid remains unchanged, and is equal to:

$$
m_{w}=m_{e}+m_{c s}+m_{h}+m_{k}+m_{r}
$$

Using the Mendeleev-Clapeyron equation for an ideal process, and the assumption that the process is isobaric, we get: 


$$
m_{w}=\frac{p V_{e}}{R_{\text {gas }} T_{h}}+\frac{p V_{c s}}{R_{\text {gas }} T_{k}}+\frac{p V_{h}}{R_{\text {gas }} T_{h}}+\frac{p V_{k}}{R_{\text {gas }} T_{k}}+\frac{p V_{r}}{R_{\text {gas }} T_{r}}
$$
fluid.

where $p$ is the pressure in the working spaces, $R_{\text {gas }}$ is the gas constant of the working

Expressing the pressure value from equation (2), we get:

$$
p=\frac{m_{w} \cdot R_{g a s}}{\frac{V_{e}}{T_{h}}+\frac{V_{h}}{T_{h}}+\frac{V_{r}}{T_{r}}+\frac{V_{k}}{T_{k}}+\frac{V_{c s}}{T_{k}}}
$$

The temperature of the regenerator is assumed constant and is calculated from the condition of linear distribution of the temperature of the regenerator along its length:

$$
T_{r}=\frac{T_{h}-T_{k}}{\ln \left(\frac{T_{h}}{T_{k}}\right)}
$$

Newton's second law (second-order differential equation) describes the movement of the working piston and displacer. For the engine configuration used in this work, the damping force on the displacer is negligible, and the resulting force is determined from the pressures in the buffer, expansion and compression spaces. The initial position of the displacer is set using the value of the initial phase shift (60 degrees) relative to the working piston; the positive direction of movement is towards the expansion space. The displacer movement is represented by the equation (5):

$$
m_{d} \frac{d^{2} x_{d}}{d t^{2}}+k_{d} x_{d}=p_{b} A_{r o d}+p_{c s} A_{p}-p_{e} A_{d}
$$

where $m_{d}$ is the mass of the displacer, $k_{d}$ is the stiffness of the displacer spring, $A_{r o d}$ is the cross-sectional area of the rod, $A_{p}$ is the cross-sectional area of the working piston, $A_{d}$ is the cross-sectional area of the displacer.

The coefficient of stiffness of the spring for the working piston is negligible for this configuration, and it's damping coefficient $D_{p}$ depends mainly on the alternator, and in this work it is taken as a constant. The movement of the working piston is described by the equation:

$$
m_{p} \frac{d^{2} x_{p}}{d t^{2}}+D_{p} \frac{d x_{p}}{d t}=\left(p_{b}-p_{c s}\right) A_{p}
$$

\subsubsection{Dynamic model}

Based on the above-described mathematical model, a dynamic model was built in the Scilab/Xcos software environment [16]. Figure 2 shows a submodel of the Stirling engine (the top-level model of the power plant will be presented later). 


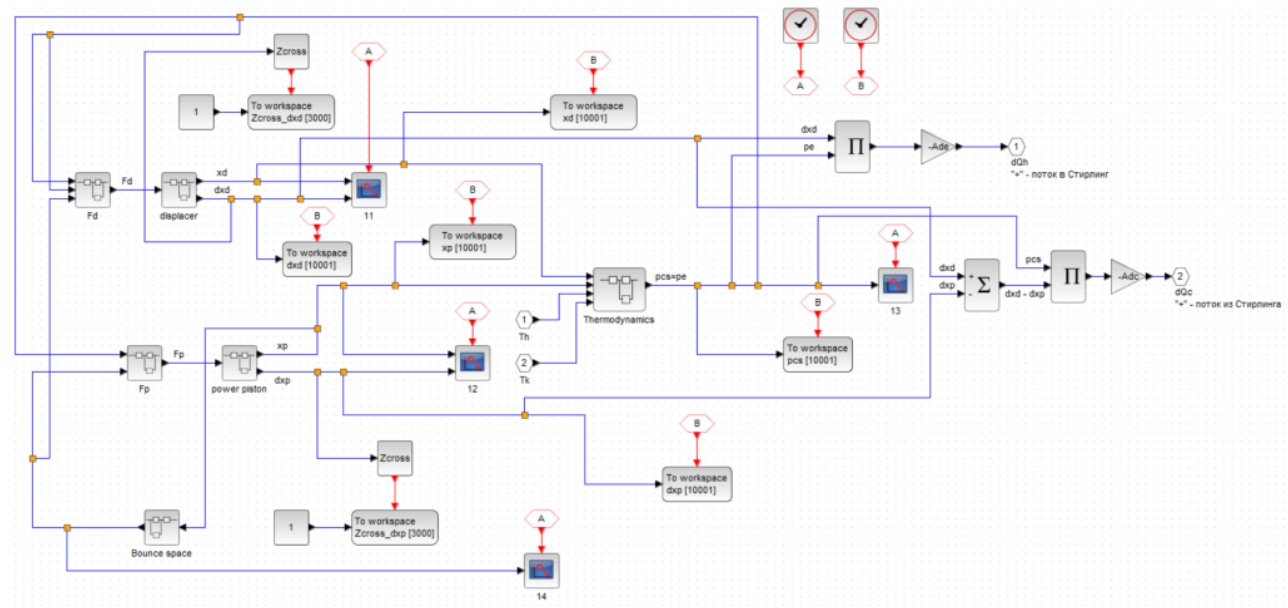

Fig. 2. Dynamic model of the Stirling engine.

Figure 3 shows a sub model for calculating thermodynamic parameters - pressure in the working cavity, and temperature of the regenerator.

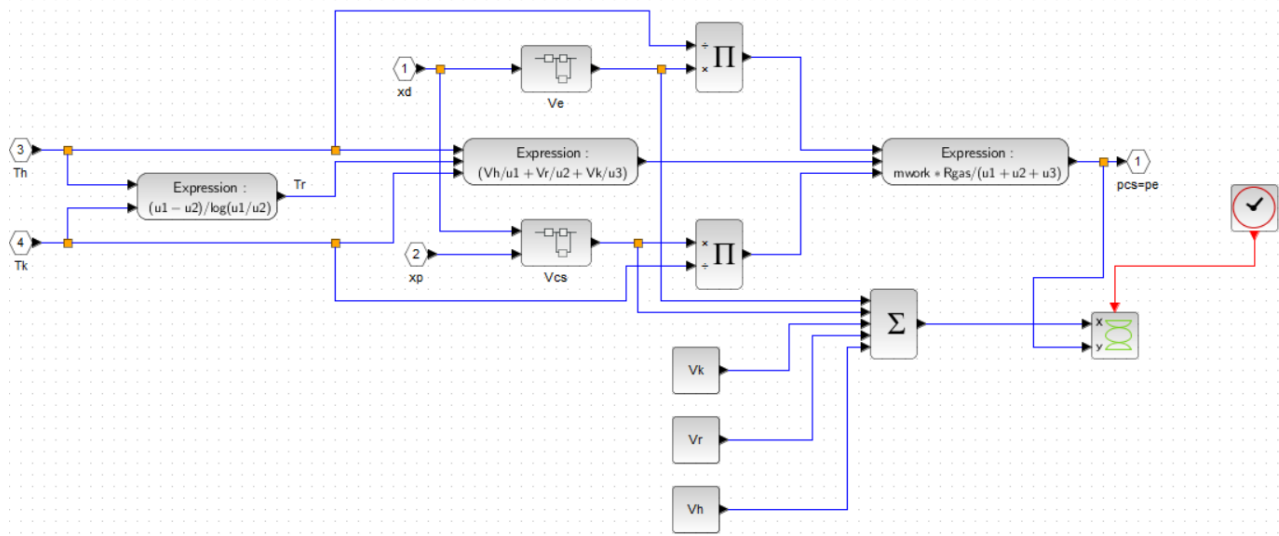

Fig. 3. Sub model for calculating thermodynamic parameters.

Figures 4 - 5 show sub models for determining the coordinates and speed of the displacer and the working piston, respectively. 


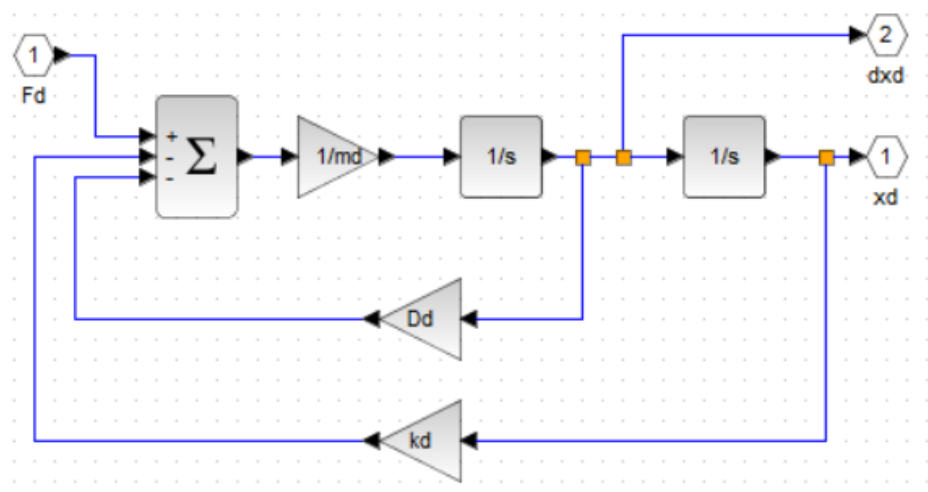

Fig. 4. Sub model for determining the position and velocity of the displacer.

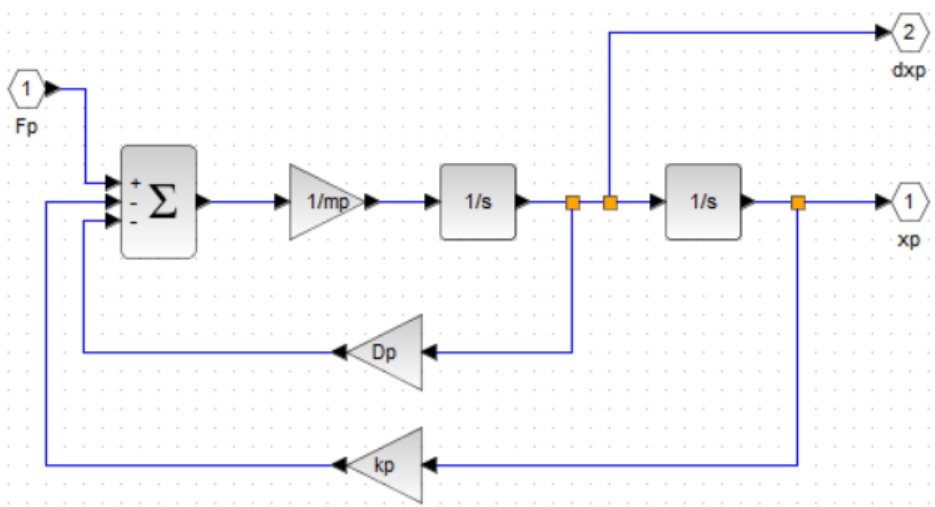

Fig. 5. Sub-model for determining the coordinates and speed of the working piston.

\subsection{Power plant model}

For the computational analysis of the power plant as a whole, a dynamic model was used, consisting of a Stirling engine model and an external thermal circuit consisting of heat pipes, heat exchangers and radiator cooling panels. The heat carrier is an alloy of potassium (77\%) with sodium $(23 \%)$. The design scheme of the installation is shown in Figure 6. A similar construction of the heat supply / removal subsystem for spacecraft is considered, for example, in $[13,17]$. 


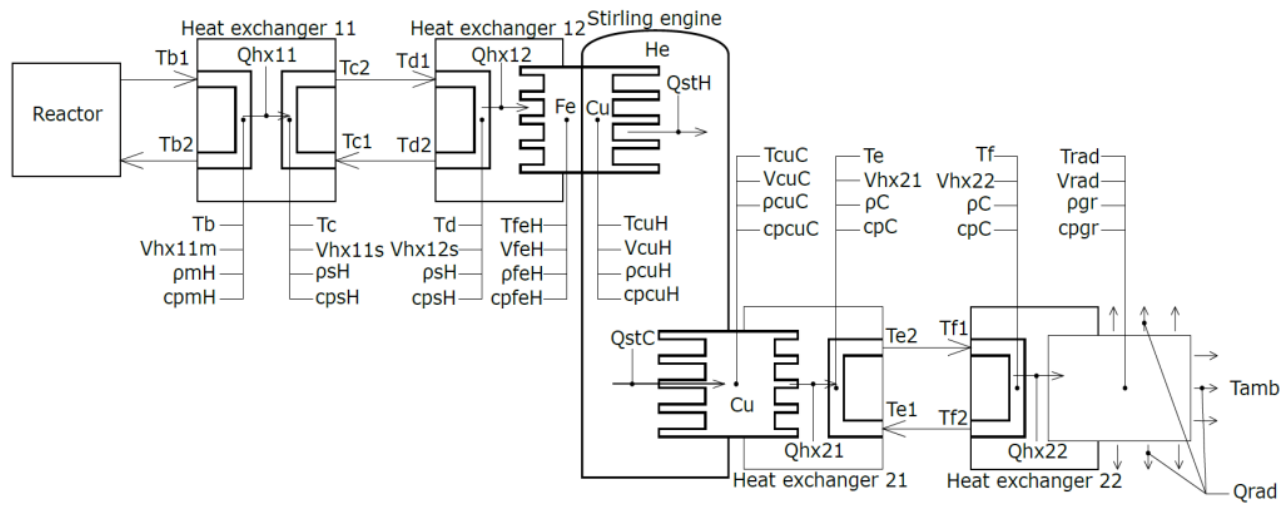

Fig. 6. Design diagram of the power plant.

\subsubsection{Mathematical model}

When formulating the mathematical model of the external heat circuit, the following assumptions were made:

- the nuclear reactor is replaced by a constant temperature source;

- heat and pressure losses during the flow of the coolant through the pipes between the heat exchangers are not taken into account;

The coolant temperature in the heating circuits was modeled with a time delay. The temperature of the heat carrier flowing into the heat exchanger 12 becomes equal to the temperature of the heat carrier flowing out of the heat exchanger 11 after a period of time required to flow from one heat exchanger to another. This effect was modeled using the following equation:

$$
\frac{d T_{d 1}}{d t}=\frac{T_{c 2}-T_{d 1}}{\tau}
$$

where $T_{d l}$ is the temperature of the coolant at the inlet to the heat exchanger 11, $T_{c 2}$ is the temperature of the coolant at the outlet of the heat exchanger $11, \tau$ is the time delay.

In addition, Xcos provides a time delay tool that accurately models the time delay by holding the values constant and outputting them later at a specific time $\tau$. All other sections of the thermal circuit were modelled in the same way.

Figure 7 shows a design scheme of heat exchanger 11 .

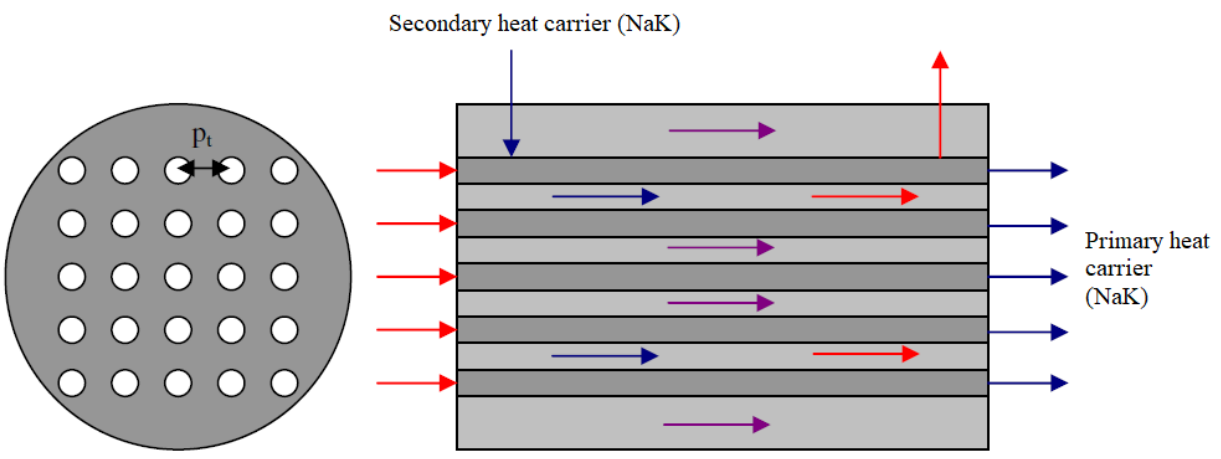

Fig. 7. Shell and tube heat exchanger with tube pitch $p_{t}$. 
The heat flow between the primary and secondary coolants in the heat exchanger 11 was modelled using the following equation:

$$
Q_{h \times 11}=\frac{\Delta T_{l m 11}}{\frac{1}{\alpha_{h x 11 m} \cdot A_{h x 11 m}}+\frac{\ln \left(d_{o} / d_{i}\right)}{2 \cdot \pi \cdot \lambda_{t} \cdot L_{t} \cdot N_{t}}+\frac{1}{\alpha_{h x 11 s} \cdot A_{h x 11 s}}}
$$

where $\Delta T_{l m 12}$ is the logarithmic temperature difference in the heat exchanger 11, $\alpha_{h \times 11 m}$ is the heat transfer coefficient of the primary coolant, $A_{h \times 11 m}=\pi d_{i} L_{t} N_{t}$ is the heat transfer area of the primary coolant, $d_{o}$ is the outer diameter of the heat exchanger tube, $d_{i}$ is the inner diameter of the tube, $\lambda_{t}$ is the heat transfer coefficient of the tube, $L_{t}$ is the tube length, $N_{t}$ is the number of tubes in the heat exchanger, $\alpha_{h \times 11 s}$ is the heat transfer coefficient secondary coolant, $A_{h x 11 s}$ is the heat exchange area of the secondary coolant.

Since the temperature of the primary and secondary heat transfer medium varies along the length of the heat exchanger, the logarithmic difference in average temperatures was used to determine the heat transfer rate, which was determined by the following equation:

$$
\Delta T_{l m 11}=\frac{\left(T_{b 1}-T_{c 2}\right)-\left(T_{b 2}-T_{c 1}\right)}{\ln \left(\frac{T_{b 1}-T_{c 2}}{T_{b 2}-T_{c 1}}\right)}
$$

The calculation equations for determining the different temperatures of the thermal circuit for hot and cold heat exchangers are given below.

\section{Hot heat exchanger:}

- Primary coolant temperature in the heat exchanger 11:

$$
\frac{d T_{b}}{d t}=\frac{1}{\rho_{m} V_{h x 11 m} c_{p m}}\left(\frac{d m_{m}}{d t} c_{p m}\left(T_{b 1}-T_{b 2}\right)-Q_{h x 11}\right)
$$

- Secondary coolant temperature in the heat exchanger 11:

$$
\frac{d T_{c}}{d t}=\frac{1}{\rho_{s} V_{h x 11 s} c_{p s}}\left(Q_{h \times 11}-\frac{d m_{s}}{d t} c_{p s}\left(T_{b 2}-T_{b 1}\right)\right)
$$

- Secondary coolant temperature in the heat exchanger 12 :

$$
\frac{d T_{d}}{d t}=\frac{1}{\rho_{s} V_{h \times 12 s} c_{p s}}\left(\frac{d m_{s}}{d t} c_{p s}\left(T_{d 1}-T_{d 2}\right)-Q_{h x 12}\right)
$$

- Heat flow in heat exchanger 12:

$$
Q_{h \times 12}=\alpha_{h \times 12 s} A_{h \times 12 s} \frac{T_{d 1}-T_{d 2}}{\ln \left(\frac{T_{d 1}-T_{m e}}{T_{d 2}-T_{m e}}\right)}
$$

- The temperature of the metal fins in the heat exchanger 12 (heat supply head of the

Stirling engine): 


$$
\frac{d T_{\text {meH }}}{d t}=\frac{1}{\rho_{\text {meH }} V_{\text {meH }} c_{\text {pmeH }}}\left(Q_{h \times 12}-Q_{S t H}\right)
$$

\section{Cold heat exchanger}

- Temperature of the refrigerator in the Stirling Engine (heat exchanger 21):

$$
\frac{d T_{\text {meC }}}{d t}=\frac{1}{\rho_{\text {meC }} V_{\text {meC }} c_{\text {pmeC }}}\left(Q_{S t C}-Q_{h x 21}\right)
$$

- Heat flow in heat exchanger 21:

$$
Q_{h \times 21}=\alpha_{h \times 21} A_{h \times 21}\left(T_{m e C}-T_{e}\right)
$$

- Heat carrier temperature in the heat exchanger 21:

$$
\frac{d T_{e}}{d t}=\frac{1}{\rho_{C} V_{h x 21 C} c_{p C}}\left(Q_{h x 21}-\frac{d m_{N a K}}{d t} c_{p C}\left(T_{e 2}-T_{e 1}\right)\right)
$$

- Heat carrier temperature in the heat exchanger 22:

$$
\frac{d T_{f}}{d t}=\frac{1}{\rho_{C} V_{h \times 22} c_{p C}}\left(\frac{d m_{N a K}}{d t} c_{p C}\left(T_{f 1}-T_{f 2}\right)-Q_{22}\right)
$$

- Heat flow in heat exchanger 22:

$$
Q_{h \times 22}=\frac{T_{f}-T_{r a d}}{\frac{1}{\alpha_{h x 22} A_{h \times 22}}+\frac{\ln \left(\frac{d_{o 22}}{d_{i 22}}\right)}{2 \pi \lambda_{p a n} N_{t u b} W_{r a d} N_{r a d}}}
$$

- Radiator panel temperature:

$$
\frac{d T_{r a d}}{d t}=\frac{1}{m_{r a d} \cdot c_{g r}}\left(Q_{h x 22}-\varepsilon \cdot \sigma \cdot A_{r a d} \cdot\left(T_{r a d}^{4}-T_{a m b}^{4}\right)\right)
$$

\subsubsection{Dynamic model}

According to the corresponding mathematical model, a dynamic model was built in the Scilab/Xcos software environment [16]. The figures below show the implementation of the model. 


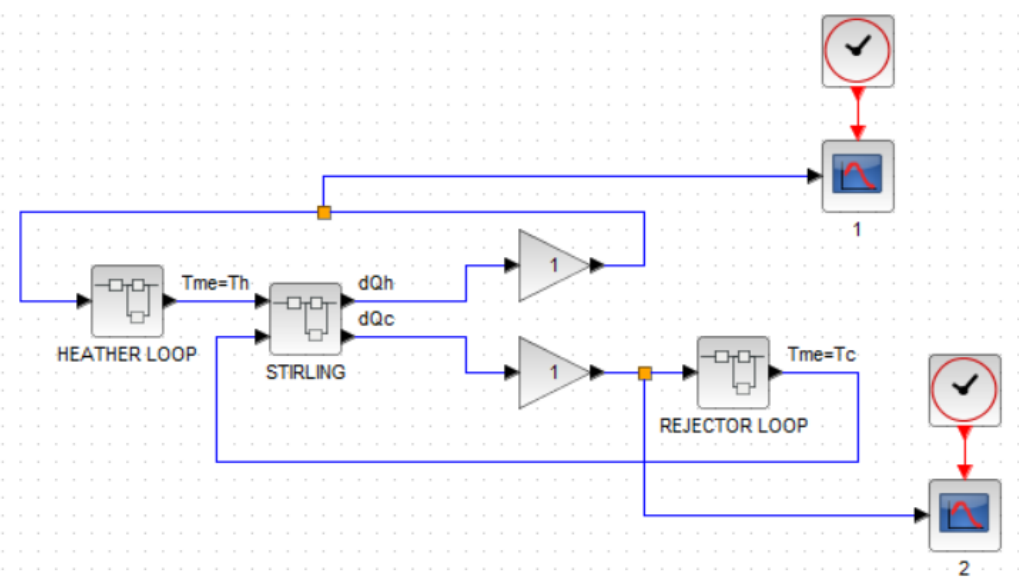

Fig. 8. Power plan top-level model.

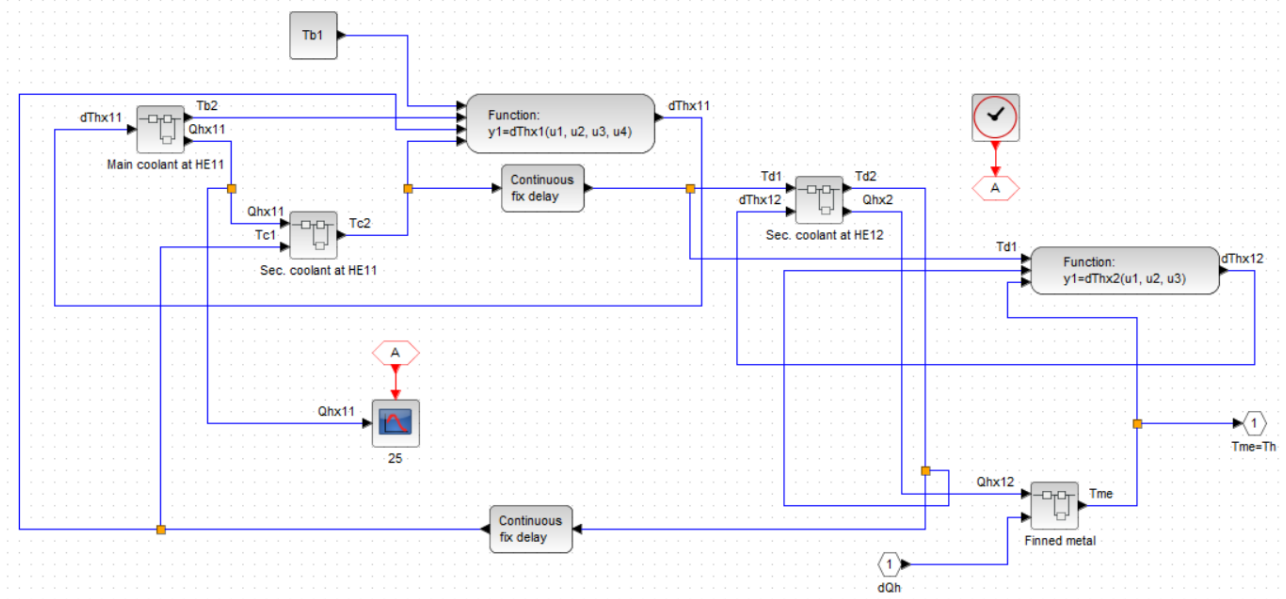

Fig. 9. Hot circuit submodel.

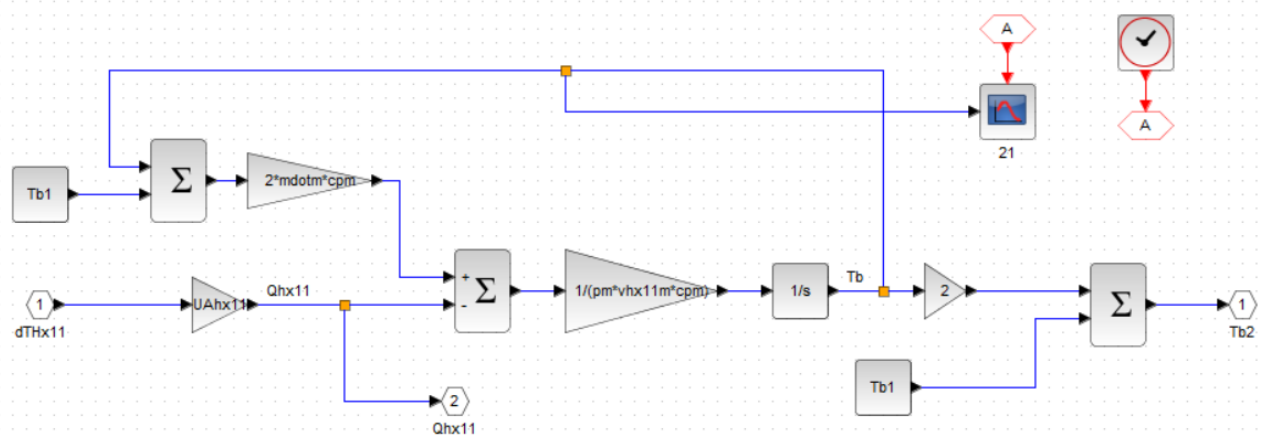

Fig. 10. Primary loop heat carrier in heat exchanger 11 Temperature $T_{b}$ detection submodel. 


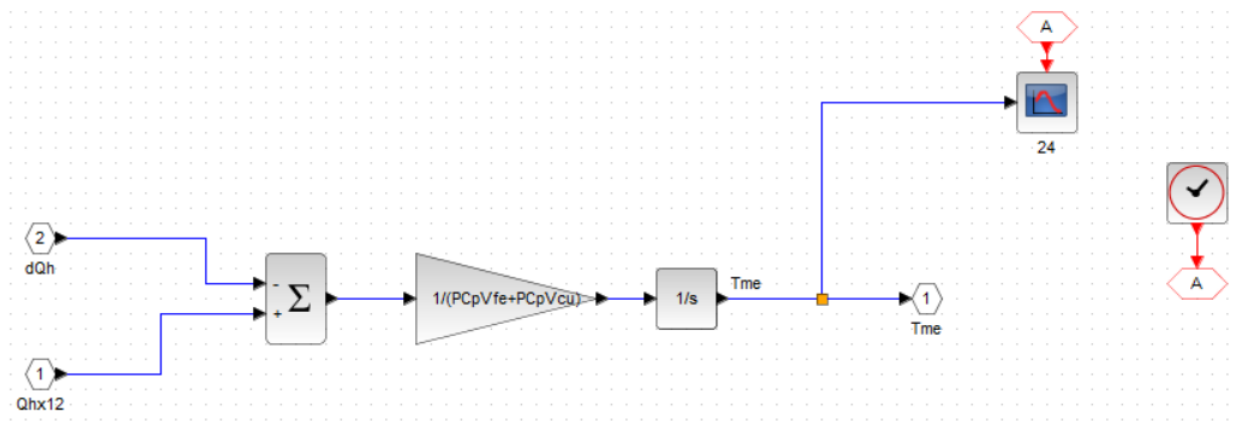

Fig. 11. Heat supply head temperature $T_{m e H}$ detection submodel.

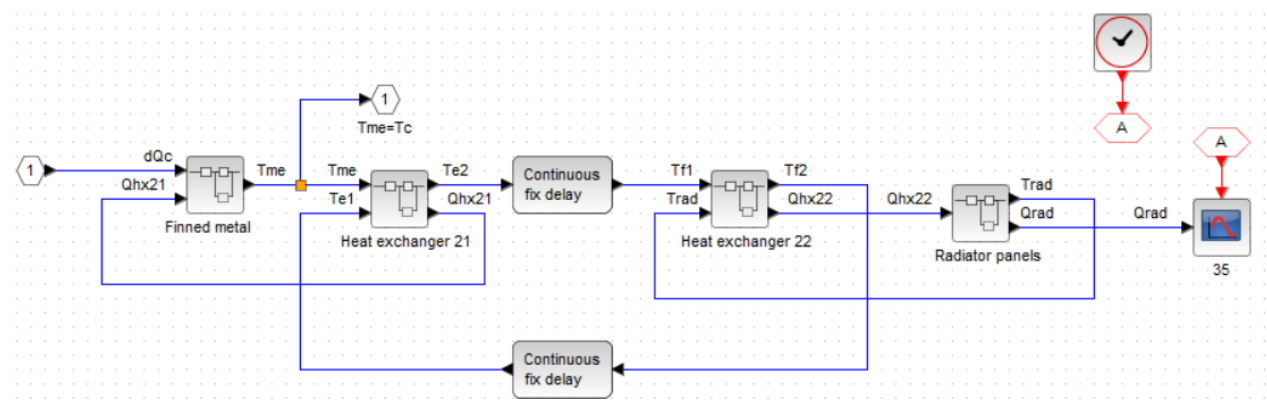

Fig. 12. Cold circuit submodel.

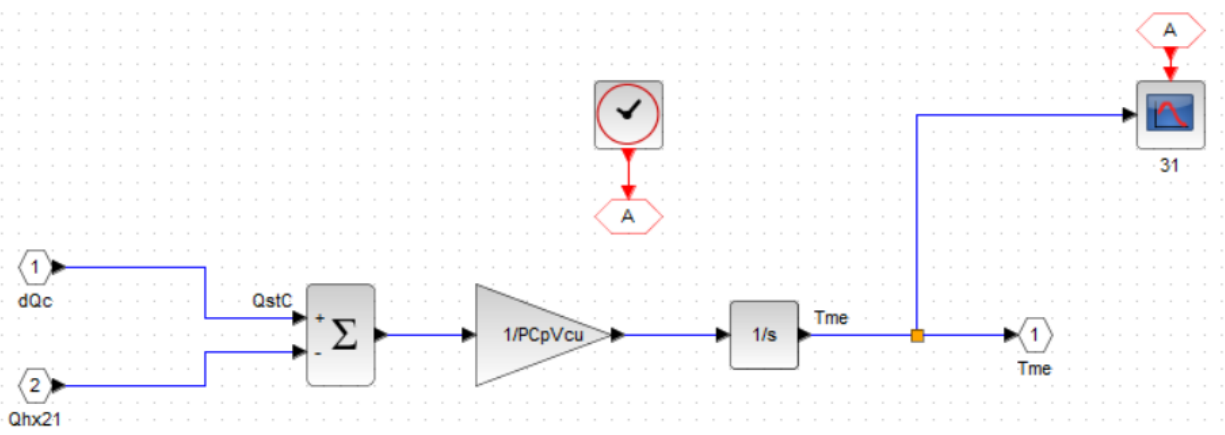

Fig. 13. Refrigerator temperature $T_{m e C}$ detection submodel. 


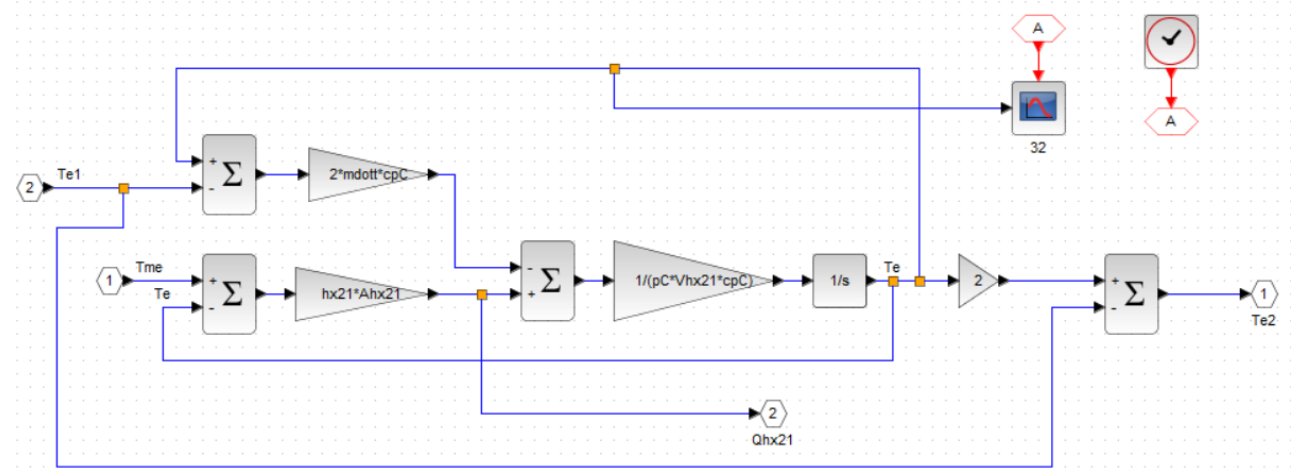

Fig. 14. Heat carrier in heat exchanger 21 temperature $T_{e}$ detection submodel.

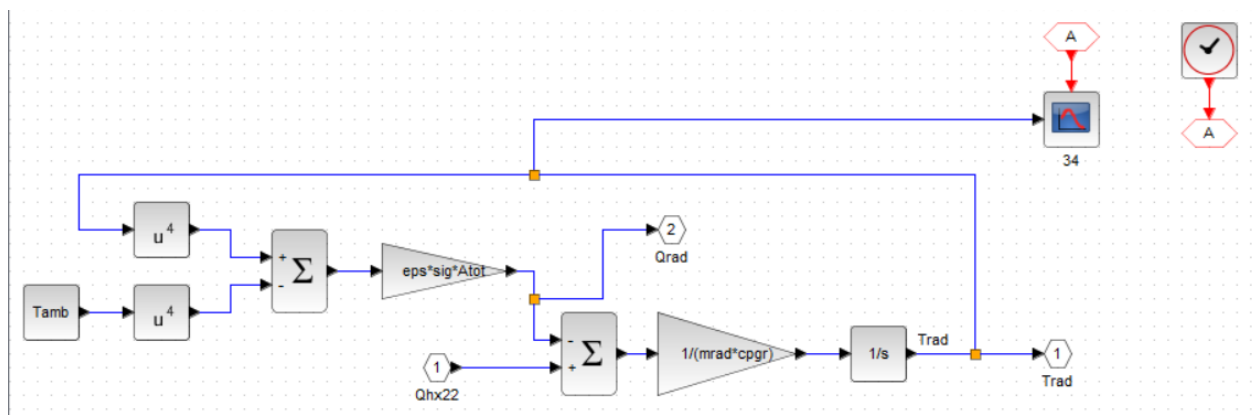

Fig. 15. Radiator temperature $T_{\text {rad }}$ detection submodel.

\section{Results}

For the calculated analysis of the power plant, a Stirling engine with a power of $N=6000$ $\mathrm{W}$ was used, at an operating frequency of $f=60 \mathrm{~Hz}$. The simulation time is 100 seconds. Reactor temperature $T_{b l}=1000 \mathrm{~K}$, ambient temperature $T_{a m b}=4 \mathrm{~K}$. Initial conditions: for hot circuit $T_{b 0}=T_{c 0}=T_{d 0}=T_{F e H}=T_{C u H}=900 \mathrm{~K}$; for cold circuit $T_{C u C}=T_{e 0}=T_{f 0}=T_{r a d}=$ $400 \mathrm{~K}$.

The figures below show the simulation results:

- Dependence of the temperature $T_{c}$ of the coolant of the secondary circuit in heat exchanger 11 on time - figure 16;

- Dependence of the temperature of the metal fins in heat exchanger 12 (heat supply head of the Stirling engine) on time, which also equal to the temperature of the working fluid in hot heat exchanger - figure 17;

- Dependence of the heat flow in the heat exchanger $11 Q_{h x l l}$ on time - figure 18;

- Dependence of the temperature of the refrigerator in the Stirling Engine (heat exchanger 21) on time, which also equal to the temperature of the working fluid in cold heat exchanger - figure 19;

- Dependence of the temperature of the coolant in heat exchanger $22 T_{f}$ on time figure 20 ;

- The dependence of the heat flow into the surrounding space $Q_{\text {rad }}$ on time - figure 21 . 
- The indicator diagram (dependence of the working fluid pressure on total volume of the working fluid - figure 22. The arrow shows the direction of the diagram displacement during heating up.

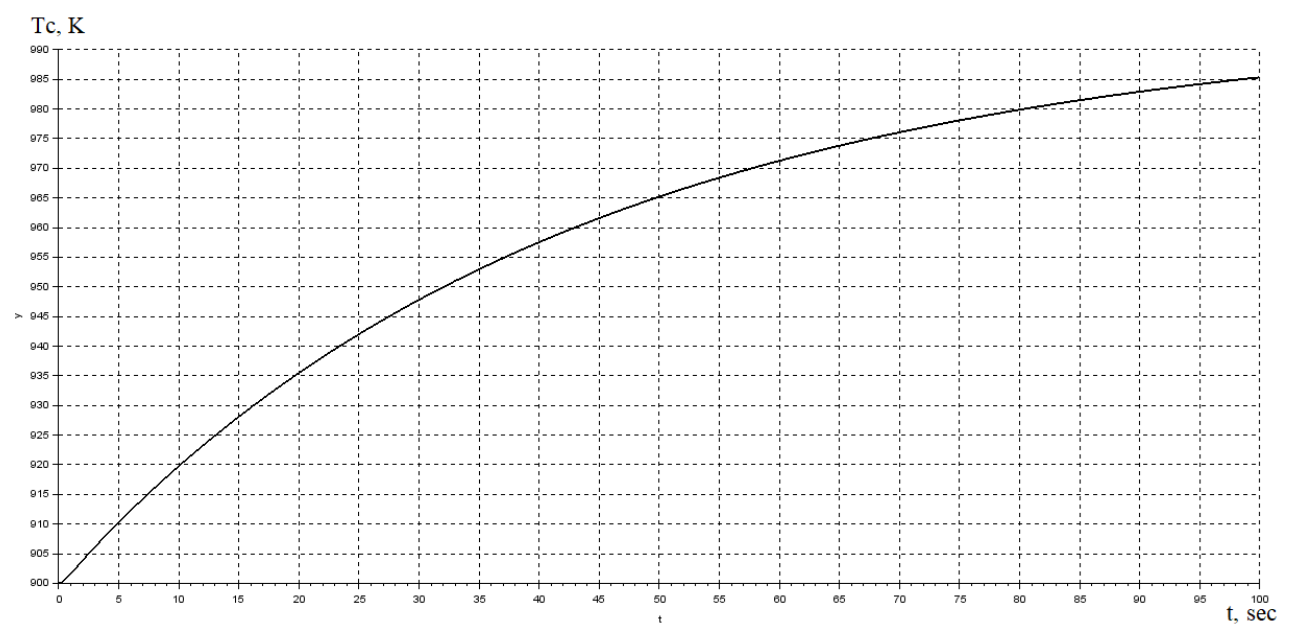

Fig. 16. The dependence of temperature $T_{c}$ on time.

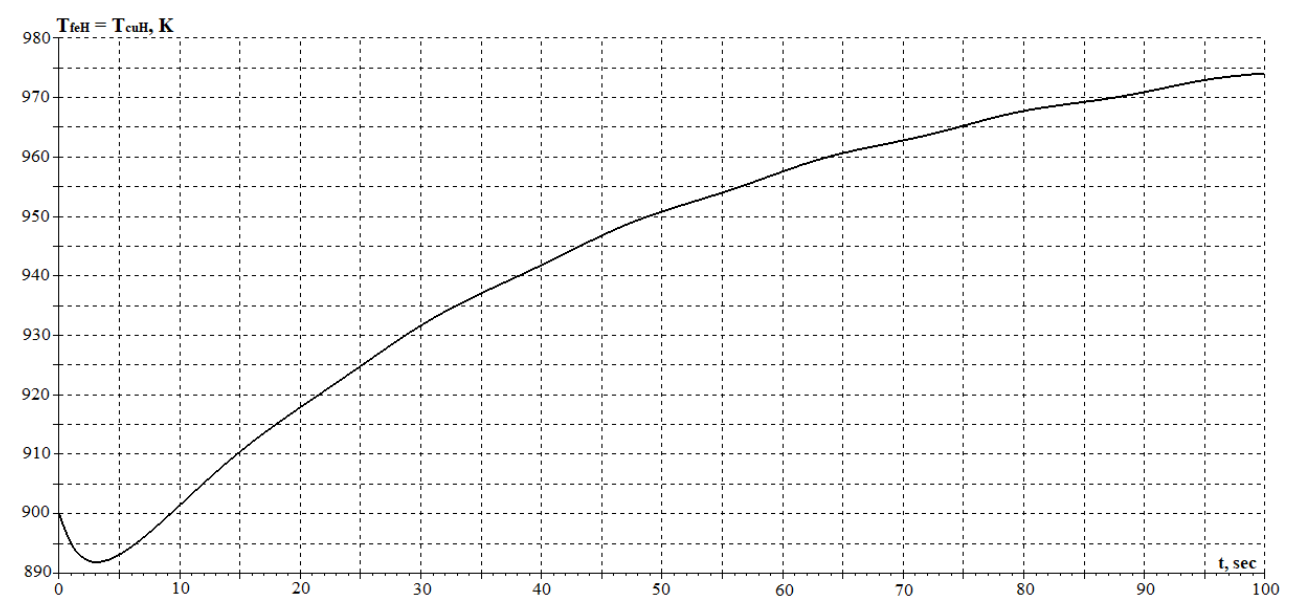

Fig. 17. The dependence of temperature $T_{\mathrm{FeH}}=T_{\mathrm{CuH}}$ on time. 


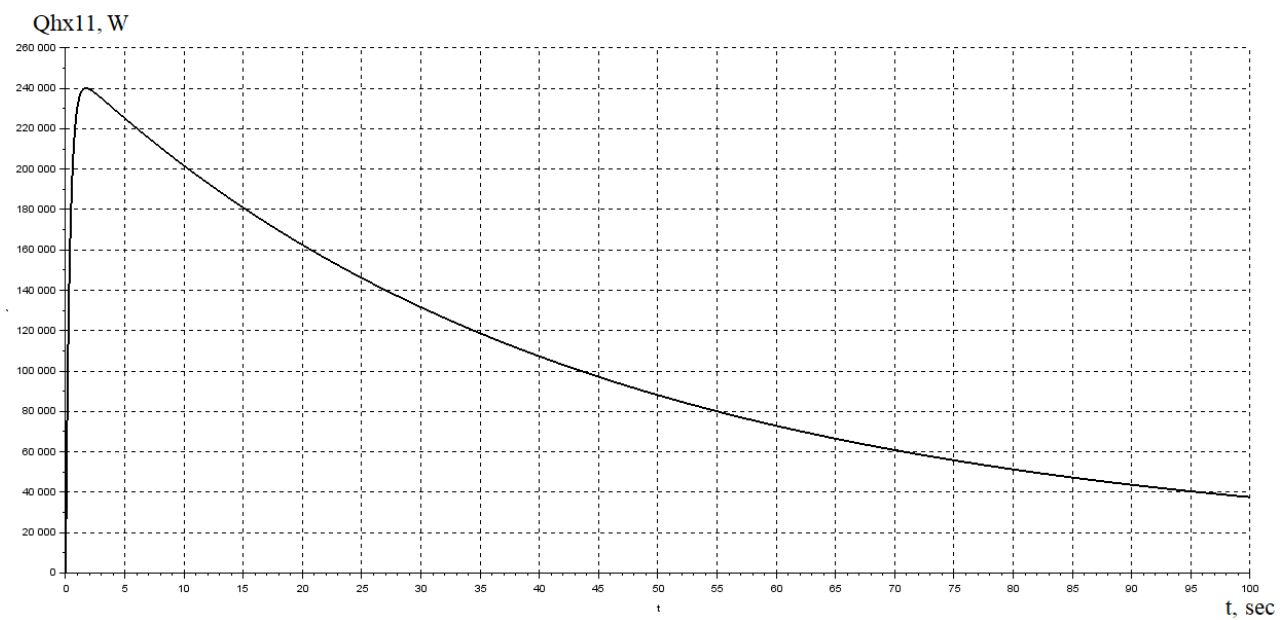

Fig. 18. The dependence of the heat flow $Q_{h x I l}$ on time.

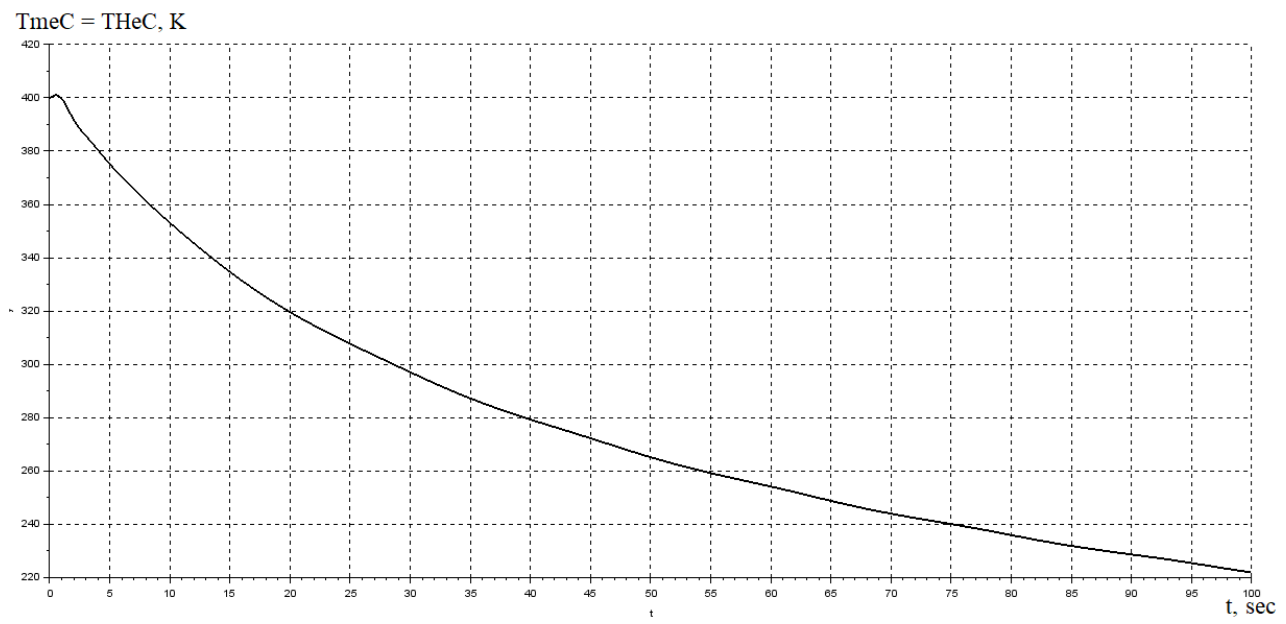

Fig. 19. The dependence of temperature $T_{m e C}$ on time. 


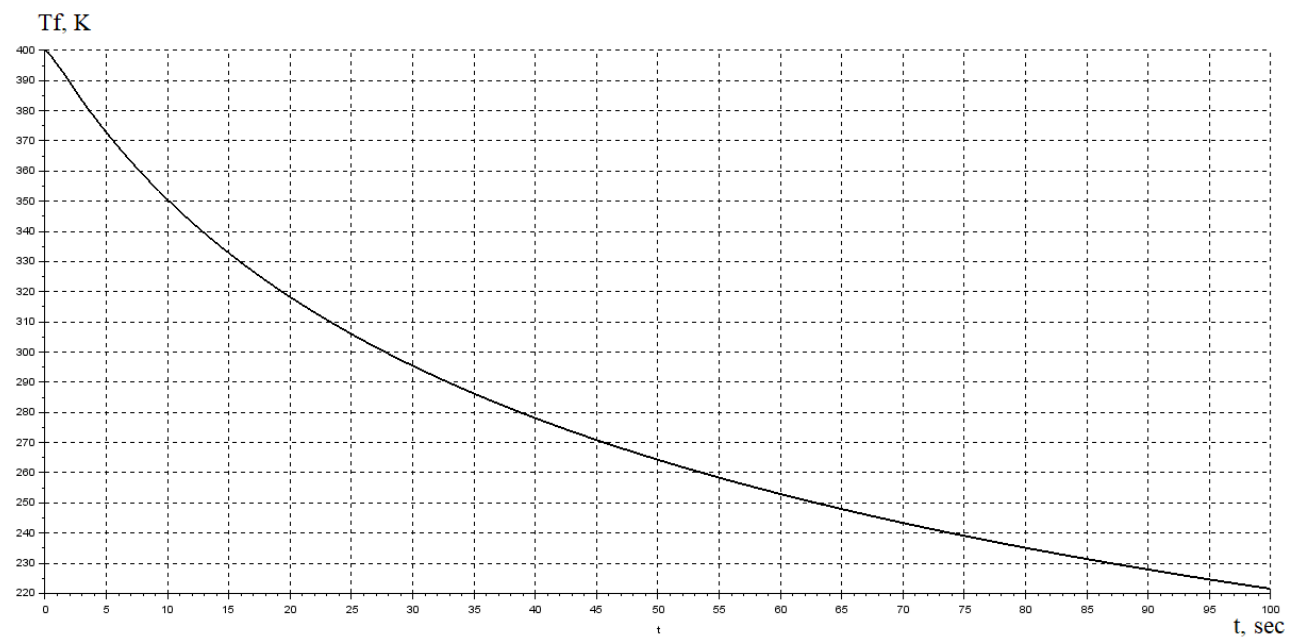

Fig. 20. The dependence of temperature $T_{f}$ on time.

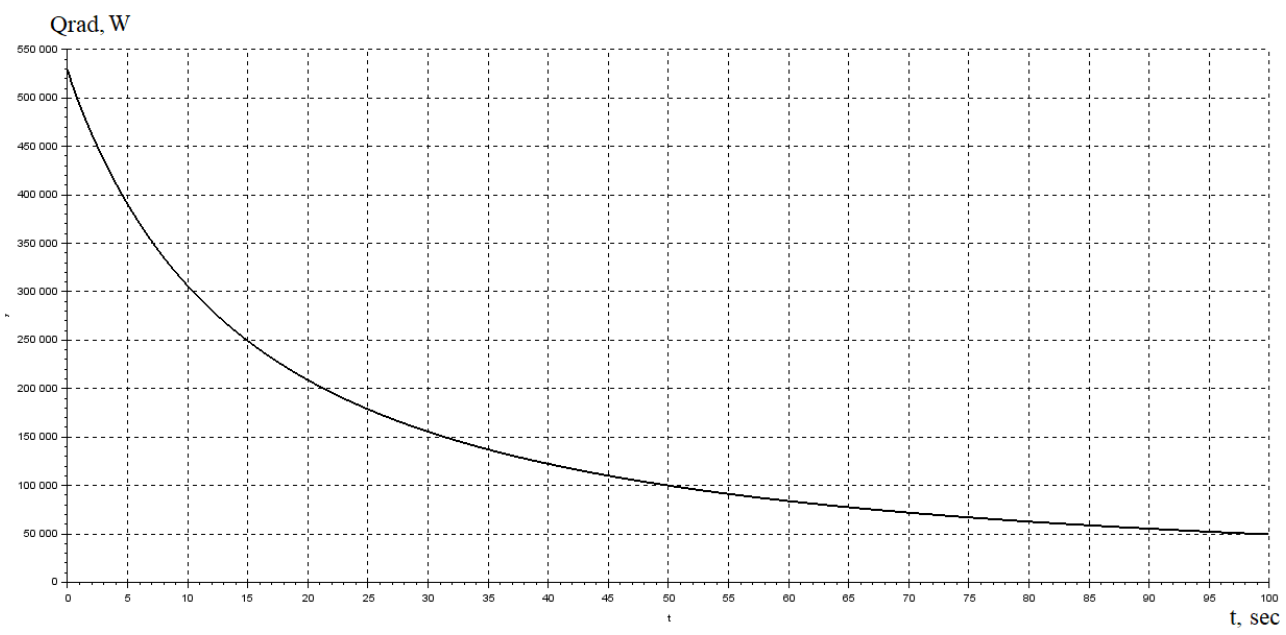

Fig. 21. The dependence of the heat flow $Q_{\text {rad }}$ on time. 


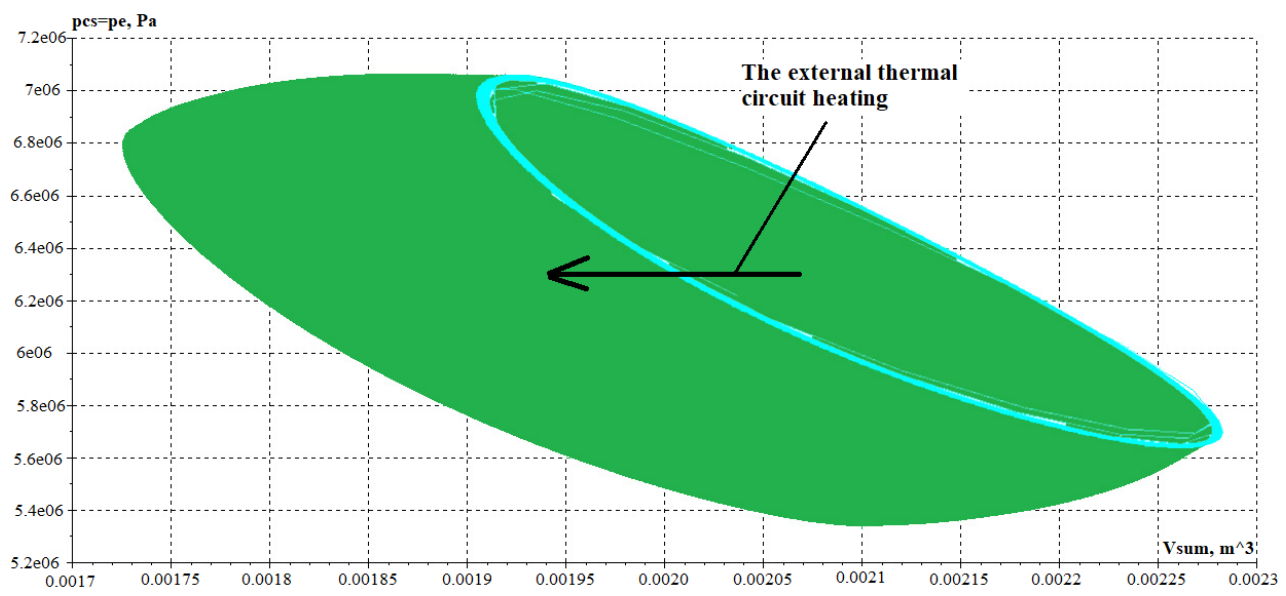

Fig. 22. The indicator diagram

\section{Conclusions}

When performing this work, the engine models and the thermal circuit were initially implemented separately, and then were combined into a single whole. A comparison of the engine simulation results with the available experimental data showed good agreement, and the thermal circuit models showed realistic behavior during warm-up.

A change in the thermal state of the circuit leads to a change in the operating temperatures of the processes inside the Stirling engine, which leads to a change in the indicator diagram; This suggests that it is possible to take into account the influence of the dynamics of the thermal circuit on the change in the performance of the engine and the power plant as a whole. The typical warm-up time of the circuit for the power plant in question is several hundred seconds. In the future, it is planned to coordinate the results of modeling the power plant with experimental data, as well as add models of a nuclear reactor and a linear generator (load).

Based on the results of the work, a dynamic model of the power plant based on the Stirling engine was created and an analysis of the heating of the external thermal circuit was carried out. This model can be used for a preliminary assessment of the main indicators of the spacecraft's power plant.

\section{References}

1. G. Walker, Stirling engines (1980).

2. W. R. Martini, Stirling engine design manual (1978)

3. V. Lyons, S. John. An Overview of Space Power Systems for NASA Missions, 5th IECEC (2007)

4. Y. V. Loktionov, L. L. Myagkov, I. V. Obolonny, A. S. Filatyev, I. A. Shirkovsky, Prospects for the use of free piston linear generating sets for energy conversion in aerospace systems, Topical issues in the design of automatic spacecraft for basic and applied research, p. 504-511 (2017)

5. Y. V. Loktionov, L. L. Myagkov, I. V. Obolonny, A. V. Syachinov, Linear FreePiston Units and New Critically Technologies for Piston Engines, Engine-2017, p. 5354 (2017) 
6. R. Dyson, S. Wilson, R. Tew, Review of computational stirling analysis methods, 2nd IECEC, p. 5582 (2004)

7. L. Daijin, K. Qin, K. Luo, Underwater striling engine design with modified onedimensional model, Int. J. Nav. Archit. Ocean Eng., 7 (2015)

8. E. Lewandowski, T. Regan, Overview of the GRC stirling convertor system dynamic model, 2nd IECEC, p. 5671 (2004)

9. S. Qui, A. Peterson, Linear Dynamic Modeling and Numerical Simulation of an STC Stirling Convertor, 1st IECEC, p. 5930 (2003)

10. T. F. Regan, S. S. Gerber, M. E. Roth, Development of a dynamic, end-to-end free piston Stirling convertor model, Expanding the Frontiers of Space, 654 (2003)

11. G. Benvenuto, F. De Monte, Electrodynamic analysis of free-piston Stirling engine/linear alternator systems, IECEC, p. 4031 (1994)

12. S. H. Zare, A. R. Shourangiz-Haghighi, A. R. Tavakolpour-Saleh, Higher order modeling of a free-piston Stirling engine: analysis and experiment, IJEEE, 9, p. 273293 (2018)

13. J. Langlois, D. Myre, M. Nelson, Dynamic computer model of a Stirling space nuclear power system, 5th IECEC, p. 4774 (2006)

14. T. Regan, E. Lewandowski, Application of the GRC stirling convertor system dynamic model, 2nd IECEC, p. 5672 (2004)

15. T. Regan , E. Lewandowski, Development of a Linear Stirling System Model with Varying Heat Input, 5th IECEC, p. 4773 (2007)

16. Scilab - open source software for numerical computation [Electronic resource]. (https://www.scilab.org/en/scilab/about).

17. C. Tarau, W. G. Anderson, K. Walker, Sodium Variable Conductance Heat Pipe for Radioisotope Stirling Systems, 7th IECEC (2009) 